\title{
A New Adaptive approach in Routing and Wavelength Assignment for Optical WDM Networks.
}

\author{
${ }^{1}$ Surendra Bhosale and ${ }^{2}$ Nisha Sarwade \\ ${ }^{1}$ Asscociate Professor, Department of Electrical Engineering,VJTI, Mumbai, India. \\ ${ }^{2}$ Asscociate Professor, Department of Electrical Engineering,VJTI, Mumbai, India.
}

\begin{abstract}
The technology employed in fiber optic communication which multiplexes a number of optical carrier signals onto a single optical fiber by using different wavelengths of laser light is known as the wavelength-division multiplexing (WDM). In optical WDM networks, when maximum flow algorithms are used for the Routing and wavelength assignment (RWA) problem, it optimizes the network resource usage. The existing routing methods rarely consider the current network load when making the routing decision. In order to overcome the drawbacks of the existing RWA methods, an adaptive routing and wavelength assignment protocol is proposed which is based on maximum flow and load balancing.

In this work, Routing and Wavelength assignment is proposed adaptively based on the type of reservation request. When a network handles an advance reservation request, the routing is done using our Max-flow algorithm and wavelength assignment is done using an advance reservation algorithm. When the network handles an immediate reservation request, routing is done using the traffic load on each link on the paths and wavelength assignment is done using an immediate reservation algorithm.
\end{abstract}

Keywords-WDM-wavelength-divisionmultiplexing, LANlocal area network, $R W A$-Routing and Wavelength assignment,NSF-National Science Foundation,Max FlowMaximum Flow, NS- Network Simulator.

\subsection{Introduction to WDM Routing:}

In fiber optic communications, the WDM is a technology which multiplexes a number of optical carrier signals onto a single optical fiber by using different wavelengths of laser light[1]. A WDM local area network (LAN) or metropolitan area network (MAN) is usually constructed based on a shared transmission media and operate in a broadcast- and-select manner, without switching (routing According to the switching technique used, a WDM WAN could be further classified as circuit-switched (wavelength routed), packet-switched, or burst-switched [15]. WDM system uses a multiplexer at the transmitter to join the signals together and a de-multiplexer at the receiver to split them apart. With the right type of fiber it is possible to have a device that does both simultaneously, and can function as an optical add-drop multiplexer. [1]

WDM technology has enabled wavelength routing, which provides the benefits of reduced switching costs as compared to high-speed electronic switches and more transparency than electronics could provide. Also WDM needs only one optical amplifier whereas Spatial Division Multiplexing (SDM) requires one amplifier per fiber. Thus, WDM technology is paving a way for the development of new services and is playing a major role in the expansion of optical networks. [3]

In WDM routing networks, the number of wavelengths available on fiber links limits the number of end-to- end connections. Moreover, a WDM network imposes additional constraints on the wavelength assignment. If switching/routing nodes are not equipped with a wavelength converter facility, then a light path must operate on the same wavelength across all fiber links that it traverses, the Routing and Wavelength Assignment (RWA) is said to satisfy the wavelength continuity constraint[13]. However, if each node is equipped with a wavelength converter, the wavelength continuity constraint disappears and the routing problem is the same as in normal circuit-switched networks where the only limiting factor is the number of available channels on each link. This continuity constraint leads to inefficient utilization of wavelength channels and results in higher blocking probability [4].

\subsection{Challenges in the WDM routing:}

The following are Challenges in the WDM routing a) As the optical packet-switched network still faces technical difficulties as the lack of optical random access memories and stringent synchronization requirements, it is not believed to be practical in the near future and little work has been devoted to multicasting in such networks [3].

b) Since a large amount of traffic can be carried via a single fiber, a single fiber failure in the system may result in disconnection of a large number of user's end-to-end connections [4].

c) Algorithms for multi-domain network where optimization is required at the border nodes are not developed [5]

d) The problem to find a path, i.e. a route, from a source to a destination node in a WDM network with a continuous and free wavelength along each link is non-trivial. Indeed, the above problem is known as the Routing and Wavelength Assignment (RWA) problem and was found to be NP-Complete [6].

e) When rerouting light paths, the number of light paths rerouted should be kept to a minimum. This leads us to consider the minimum-disruption link-disjoint paths (MDLDP) problem. The MDLDP problem is NP-complete when the primary and backup light paths use different wavelengths. However, it is polynomially solvable for the same wavelength. [7] 
Proc. of the Intl. Conf. on Advances in Computing, Control and Networking - ACCN 2015.

Copyright ( $)$ Institute of Research Engineers and Doctors, USA .All rights reserved.

ISBN: 978-1-63248-038-5 doi: 10.15224/ 978-1-63248-038-5-89

h) When minimization of the capacity utilization is the only objective, the congestion level index or the total number of capacity used in some links may reach to their upper limits, although the total number of wavelength channels used in the network is minimized [8].

i) Assigning a longer path when a shorter path is available increases the opportunity cost for the network to accept future arrivals. However, even in a network routed only by ASPF, the shortest available paths discovered for an end-to-end node pair at run time become longer when the network is congested. They show that limiting the number of hops for accepted connections effectively reduces overall blocking probability when the network load is high. However, it has a negative effect when the load is low [9].

\section{Traffic grooming in Optical Networks:}

The problem of traffic grooming, routing and wavelength assignment (GRWA) is considered with the objective of minimizing the number of transponders in the network. We first formulate the GRWA problem as an integer linear programming (ILP) problem. Unfortunately, the resulting ILP problem is usually is very hard to solve computationally, for large networks. To overcome this difficulty a decomposition method is proposed that divides the GRWA problem into two smaller problems: the traffic grooming and routing (GR) problem and the wavelength assignment (WA) problem. In the GR problem, we only consider how to groom and route traffic demands onto light paths and ignore the issue of how to assign specific wavelength to light paths. Similar to the GRWA problem, we can formulate the GR problem as an ILP problem. The size of the GR ILP problem is smaller than its corresponding GRWA ILP problem. Furthermore, we can significantly improve computational efficiency for the GR ILP problem by relaxing some of its integer constraints, which usually leads to quite good approximate solutions for the GR problem. Once we solve then consider the WA problem, in which our goal is to derive a feasible wavelength assignment solution.

\subsection{The GRWA Problem:}

An optical mesh network architecturally has two layers: a physical layer and an optical layer. The physical layer consists of fiber spans and nodes and the optical layer consists of light paths (optical links) and a subset of nodes contained in the physical layer. A light path in the optical layer is a path connecting a pair of nodes via a set of fiber spans in the physical layer. We assume that light paths and their routes in the physical layer are given [13]. We use graph $G_{f}=\left(V_{f} E\right)$ to represent the physical layer, where $\mathrm{E}$ is the set of edges representing fiber spans and $V_{f}$ is the set of nodes representing locations which are connected via fiber spans. We use graph $G_{0}=\left(V_{0}, L\right)$ to represent the optical layer, where $L$ is the set of edges representing lightpaths and $V_{0} \subset V_{f}$ is a subset of locations that are connected via light paths. Each edge in $\mathrm{L}$ corresponds to a path in $G_{f}$. Here we treat each lightpath as a logical connection between a pair of nodes. Therefore, one light path can contain multiple wavelengths. For ease of exposition, we first assume that $G_{0}$ is a directed graph.[14]

The GRWA problem can be formulated as an integer linear programming (ILP) problem. First, we need to introduce some necessary notation:

W: the set of wavelengths available on each fiber;

D: the set of traffic demands;

$g$ : the capacity of a single wavelength;

$s_{d}$ : the size of demand $d \in D$;

$A=\left[a_{\left.D_{1}\right]}\right]_{\left.\left.\mathrm{V}_{0} \mid x\right] L\right]}$ : the node-edge incidence matrix of graph $G_{0}$;

$B=\left[b_{e_{\Perp} I}\right]_{|E| \mathbb{X}|\Sigma|}$ : the fiber light path incidence matrix;

$u_{d}=\left[u_{W d}\right]_{D \in V_{0}}:$ source destination.column vector for $d \in D$;

$x_{d}=\left[x_{l d}\right]_{l \in L}:$ column vector containing lightpath routing variables for $d \in D$;

$\mathrm{w} x_{\mathbb{d}}=1$ if demand d traverses lightpath 1 , and 0 otherwise; $y_{W}=\left[Y_{L_{W}}\right]_{l \in L}$ :the column vector containing wavelength assignment variables for $w \in W$,

$1=\left[1,1_{s} 1_{s} 1_{s} \ldots \ldots\right.$ 1] :the unit column vector of appropriate size.

Then the GRWA problem can be formulated as the following ILP problem $\left(\min \mathscr{h}_{\mathrm{W} W}\right)$

$$
\begin{aligned}
& \min \sum_{W \in W_{l} l \in L} Y_{d W} \\
& \text { s.t } A x d=u_{d} \quad d \in D \\
& B y_{W} \leq 1 \quad W \in W
\end{aligned}
$$

$$
\sum_{d \in D} s_{d} x_{\mathbb{d}} \leq g \sum_{W \in W} \mathscr{Y}_{d W} \quad l \in L
$$

where the objective function $\Sigma_{W \in W_{\alpha} l \in L} \mathscr{W}_{d W}$ is the total number of wavelength assigned to all light paths.

The three constraints are: Equation (1) is the flow balance equation, which guarantees that the light paths selected based on $x_{\mathbb{d}}$ constitute a path from the starting node of $d$ to the end node of d. Equation (2) implies a single wavelength along each fiber span can be assigned to no more than one light path. Equation (3) is the capacity constraint for light path $l$, since $\sum_{d \in D} s_{d} x_{l d}$ is the total amount of demands carried by light path $l$ and $g \Sigma_{W \in W} Y_{d W}$ is the total capacity of lightpath $l$. 
Proc. of the Intl. Conf. on Advances in Computing, Control and Networking - ACCN 2015.

Copyright ( $)$ Institute of Research Engineers and Doctors, USA .All rights reserved.

ISBN: 978-1-63248-038-5 doi: 10.15224/ 978-1-63248-038-5-89

\subsection{The GR problem:}

Let $t=\left[t_{1}\right]_{i \in L}$ a column vector containing light path capacity decision variable where $t_{1}=\Sigma_{w \in W} y_{L_{L}}$ is the number of wavelengths needed for light path $l \in L$. Then, the GR problem can be formulated as

$$
\begin{aligned}
& \min _{\mathbb{W} \in L} t_{\mathbb{I}} \\
& \text { s.t. } A x d=u_{d} \quad d \in D \\
& B t \leq|W| \mathbf{1} \\
& \sum_{\mathbb{d} \in \mathbb{D}} s_{d} l_{\mathbb{l}} \leq g t_{\mathbb{I}} \quad l \in L
\end{aligned}
$$

\subsection{The WA Problem :}

The WA problem of our interest is to find a binary solution y such that

$$
\sum_{w \in W} y_{W}=t \text { and } B y_{W} \leq \mathbf{1} \text { for } w \in W
$$

where $\mathrm{t}$ is a feasible (or optimal) solution of the GR problem.

\section{Proposed Solution Wavelength Assignment in WDM Networks:}

The route, on which data transmission takes place, is assigned a wavelength using wavelength assignment. Cost gets reduced on proper assignment of wavelengths which leads to reduced or no use of wavelength converters. A request is sent to the controller, when a source node generates a call. The controller contains the information about the free and busy wavelengths at that instant of time since it has knowledge about the network. From the set of free wavelengths, a wavelength is selected by the controller and assigns it to the call. The performance of the network is affected while selecting the wavelength. First-fit wavelength assignment strategy is mostly used in the wavelength assignment strategy. The orders of wavelengths are predefined in order to implement the wavelength assignment and it maintains a list of used and free wavelengths. The connection is established using the first wavelength in the assignment scheme and only when the wavelength is free on all the links of the selected route the connection gets established.

If not, the next indexed wavelength is used for establishing connection. This process is continued up to last wavelength. The wavelength gets added to the free wavelength set when the call is completed[7].The available wavelength with the smallest index is chosen for the first fit wavelength assignment algorithm and almost the same performance as that of the other complex algorithms is achieved and the implementation is also simple.
The First-Fit wavelength assignment algorithm is applied directly to the selected route if there is no availability of wavelength converters. The route is divided into $t+1$ segments when wavelength converters $t \geqslant 1$, which is shows First-fit algorithm is applied on each segment during fullrange wavelength conversion. All the free wavelengths on each segment is checked for the limited-range wavelength conversion and a light-path satisfying the constraint of limitedrange wavelength conversion and also having the smallest index is found. [7]

A considerable communication overhead which requires a centralized network structure to be implemented becomes to be a major disadvantage in these algorithms.

The basic limitations to be followed for the purpose of the wavelength assignment are:

1. Wavelength continuity constraint: A light path having same wavelength should be used from the source to the destination edge.

2. Distinct wavelength constraint: All the light paths within a link must be assigned different wavelengths [9].

In this paper, we propose to develop an efficient wavelength assignment algorithm in addition to the maximum flow based routing algorithm developed earlier [12]. In order to support advance reservation services, we need to concentrate both on the advance and immediate reservations. In addition to advance reservation requests, the network must also handle regular immediate reservation requests, which are typically characterized by a pair of source and destination nodes without a specified duration. Here when a network handles an advance reservation request, the routing is done using MAXFLOW algorithm[12]. When the network handles an immediate reservation request, we first calculate the traffic load on each link on the paths. Then, we assign reservation protocols for the wavelength assignment. Two reservation protocols are discussed here namely: WAAR (Wavelength Assignment for Advance Reservation) and WAIR (Wavelength Assignment for Immediate Reservation).

\subsection{Routing in WDM networks:}

\subsubsection{Max Flow Algorithm}

The routing in WDM network is carried out using Max flow algorithm described in our paper [12].

\subsubsection{Load Balancing}

The load balancing of the nodes can be performed using the method described in our paper [12].

\subsection{Wavelength Assignment or Reservation Models:}

\subsubsection{WAAR (Wavelength Assignment for Advance Reservation)}

A WAAR request consists of a source node, a destination node, a bandwidth demand, a specified starting time St, and a specified duration D. Hence, the ending time $\mathrm{T}_{\text {end }}$ is $\left(S_{\mathrm{t}}+D\right)$. Since a reservation can only start at the beginning of a timeslot and the duration is an integer number of timeslots, we assume that $S_{t}=a \tau$ and $D=\mathrm{b} \tau$, where both $a$ and $b$ are a positive 
Proc. of the Intl. Conf. on Advances in Computing, Control and Networking - ACCN 2015.

Copyright $($ Institute of Research Engineers and Doctors, USA .All rights reserved.

ISBN: 978-1-63248-038-5 doi: 10.15224/ 978-1-63248-038-5-89

integer. Accordingly, the goal is to find a feasible route that can meet the specified requirements. A solution is as per Algorithm 1.

\subsubsection{WAIR (Wavelength Assignment for Immediate} Reservation)

A WAIR request consists of a source node, a destination node, a bandwidth demand, and a specified starting time $S_{t}$, while the duration $D$ is not specified. However, the users generally have a minimum duration requirement, $D_{\min }=b \min \tau$

and expect to get as long network services as possible.

We impose an upper bound Max $T_{\text {end }}$ on the maximum ending time. Accordingly, the goal is to find a feasible route that can maximize the duration within the range of

$$
\left[D_{\min }, \operatorname{Max}_{T_{\text {end }}}-S_{t}\right] \text {. }
$$

Let $D_{\max }(\alpha)$ denote the longest duration available for route $\mathrm{R} \alpha$ and set $D \max (\alpha)=0$. A solution can be described as in Algorithm 2.

\section{Algorithm 1 (WAAR)}

1. For each alternate route

$\mathrm{R}_{\mathrm{A}} l \in(\mathrm{N} \alpha, \mathrm{D} \alpha),(\alpha=1,2, \ldots ., N r)$, check the wavelength availability on each link along the route.

2. For each link $I_{(m, n)} \in D \alpha$, check the wavelength availability in each timeslot, $\mathrm{T}_{\mathrm{S}}(1) \in\left[\mathrm{T}_{\text {end }}, \mathrm{T}\right.$ end $\left.+D\right]$

3. If $W_{I(m, n)}(I)=0$ for any timeslot $T S(1) \in\left[S_{t}, S_{t}+D\right]$,

3.1 Go to step 1 Else

3.2 If An identical wavelength is present on link $I(m, n)$ for all timeslot TS(I) $\in\left[S t, S_{t}+D\right]$, then

3.2.1 If an identical wavelength is present on all links along the route during $\left[\mathrm{T}_{\text {end }}, \mathrm{T}_{\text {end }}+\mathrm{D}\right]$.

3.2.1.1Any one identical wavelength is available, then one of the available wavelengths is selected and is reserved on each link along the route during

$$
\left[\mathrm{T}_{\text {end }}, \mathrm{T}_{\text {end }}+\mathrm{D}\right]
$$$$
\text { Else }
$$

3.2 .2 Go to step 1

$$
\text { End if }
$$

Else

3.3 Go to step 1

End if

End if

4. If a feasible route is found, then

4.1 Stop

Else

4.2 Request gets blocked.

\section{Algorithm 2 (WAIR)}

1. For each route $R_{\alpha} \in R=\left(N_{\alpha}, D_{\alpha}\right)$, $(\alpha=1,2, \ldots ., N r)$, check the longest duration available.

2. For each possible duration $D=b \tau,(b=$ $\left.b_{\min }, \ldots\left(D_{\max }-\alpha\right)\right)$, check the wavelength availability on each link along the route.

3. For each link $I(m, n) \in D(\alpha)$, examine the wavelength availability in each timeslot $T S(1) \in\left[S_{t}, S_{t}+D\right]$

4. If $W_{I}(m, n)(I)=0$ for any timeslot $T S(I) \in[S t, S t+D]$

4.1 Go to step 1 Else

4.2 If An identical wavelength is present on link $l(m, n)$ for all timeslots, $T S(l) \in\left[S_{t}, S_{t}+D\right]$, then 4.2.1 Check if an identical wavelength is present on all links along the route during $\left[\mathrm{T}_{\text {end }}, \mathrm{T}_{\text {end }}+\right.$ D] .

4.2.1.1 Anyone identical wavelength is available, let $D_{\max }(\alpha)=D$ and go to step 2 .

Else

End if 4.2.2 Go to step 1 .

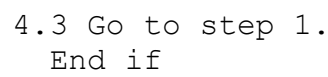

5. After all alternate routes have been examined, determine the available longest duration, i.e. $D_{\max }=\operatorname{Max}\left[D_{\max }(\alpha) ; \alpha=1,2, \ldots, N r\right]$.

6. If $D_{\max } \neq 0$, then

6.1 The route that provides the longest duration is the desired route. Else

6.2 The request gets blocked.

\subsection{Overall Algorithm}

1. Initially, we check the type of RWA reservation type whether it is Advance or Immediate reservation.

2. If reservation type is advance, then

2.1 Routing is processed according to the Max flow algorithm described in [12].

2.2 Wavelength assignment is performed as per WAAR algorithm.

3. Else if reservation type is immediate, then

3.1 Load balanced routing is performed according to [12]

3. 2 Wavelength assignment is performed as per WAIR algorithm

End if 
Proc. of the Intl. Conf. on Advances in Computing, Control and Networking - ACCN 2015.

Copyright ( $)$ Institute of Research Engineers and Doctors, USA .All rights reserved.

ISBN: 978-1-63248-038-5 doi: 10.15224/ 978-1-63248-038-5-89

\section{Simulation Results}

\subsection{Simulation Settings}

In this section, we examine the performance of our New Adaptive Routing and Wavelength Assignment (ARWA) Protocol with an extensive simulation study based upon the NS-2 network simulator [2]. We use the Optical WDM network simulator (OWNs) patch in NS-2, to simulate a NSF network (Figure.1) of 14 nodes.

In this simulation, a dynamic traffic model is used, in which connection requests arrive at the network according to an exponential process with an arrival rate $\mathrm{r}$ (call/seconds). The session holding time is exponentially distributed with mean holding time s (seconds). The connection requests are distributed randomly on all the network nodes. In all the experiments, the results of our proposed .New Adaptive Routing and Wavelength Assignment (ARWA) Protocol is compared with the TLSDRAW Based Routing and wavelength assignment (Tradeoff-LSDRAW) protocol [11].

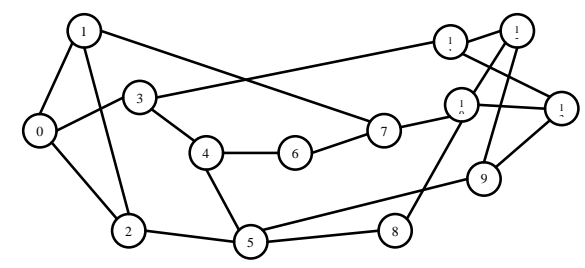

Figur 1: NSF network with 14 nodes.

\begin{tabular}{|l|l|}
\hline Topology & Mesh \\
\hline Total no. of nodes & 14 \\
\hline Link Bandwidth & $50 \mathrm{Mb}$ \\
\hline Link Wavelength Number & 10 to 25 \\
\hline Link Delay & $20 \mathrm{~ms}$ \\
\hline Link Utilization sample Interval & 0.5 \\
\hline Traffic Type & Exponential \\
\hline Traffic Arrival Rate & 0.2 \\
\hline Traffic Holding Time & 0.5 \\
\hline Traffic Packet Rate & $5 \mathrm{Mb}$ \\
\hline Packet Size & 200 \\
\hline No. of Session-traffics & 1 to 5 \\
\hline Maximum Requests & 50 \\
\hline
\end{tabular}

Table 1: Simulation Parameters

\subsection{Performance Metrics:}

In this simulation, the bandwidth utilization, end-to-end delay and throughput are measured. Bandwidth Utilization is the ratio of bandwidth received into total available bandwidth for a traffic flow. The end-to-end-delay is averaged over all surviving data packets from the sources to the destinations. Throughput is the number of packets received successfully.

\subsection{Results}

\section{A. Effects of Varying Flow:}

In the initial simulation, the traffic flow is varied as $1,2,3,4$ and 5 and measure the throughput, end-to-end delay and channel utilization.

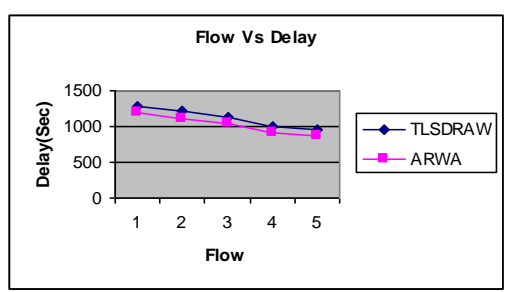

Fig 2: Flow Vs Delay

(The end-to-end delay occurred when the rate is increased.)

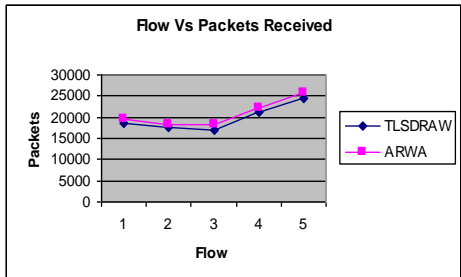

Fig 3: Flow Vs Packets Received

(The throughput occurred when the rate is increased.)

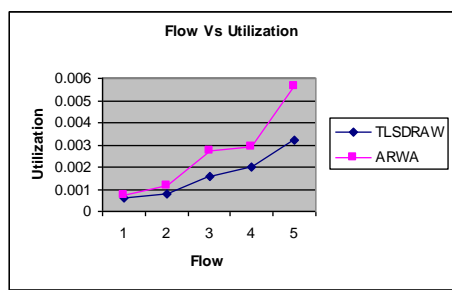

Fig 4: Flow Vs Utilization

(The channel utilization improved when the rate is increased)

\section{B. Effect of Varying Wavelength:}

In this experiment, the link wavelength is varied as 10, 15, 20 and 25 and measure the throughput, end-to-end delay and channel utilization.

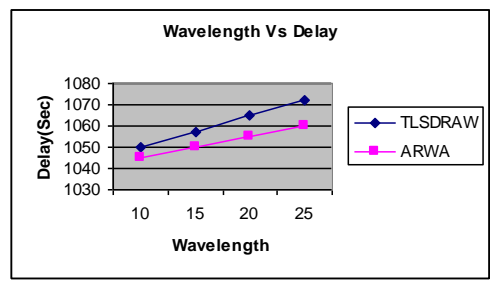

Fig 5: Wavelength Vs Delay

(The end-to-end delay occurred when the no. of flows is increased.).

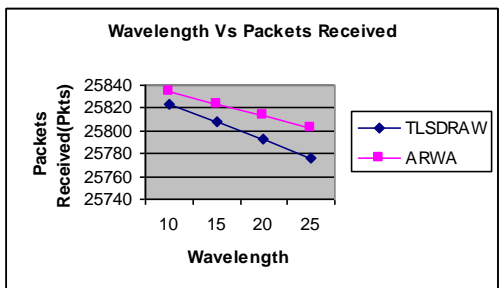

Fig 6: Wavelength Vs Packets Received

(The throughput occurred when the no. of flow is increased.) 
Proc. of the Intl. Conf. on Advances in Computing, Control and Networking - ACCN 2015.

Copyright ( $)$ Institute of Research Engineers and Doctors, USA .All rights reserved.

ISBN: 978-1-63248-038-5 doi: 10.15224/ 978-1-63248-038-5-89

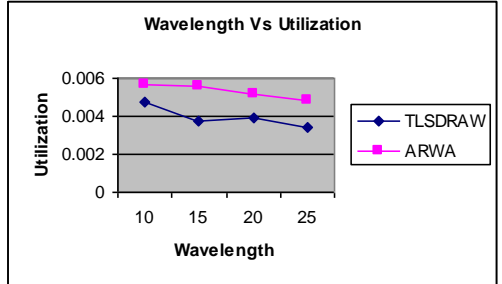

Fig 7: Wavelength Vs Utilization

(The channel utilization obtained when the no. of flows is increased. )

\section{Conclusion}

In this paper, we have developed a new adaptive routing and wavelength assignment protocol which is based on maximum flow and load balancing. In order to support advance reservation services, we need to concentrate both on the advance and immediate reservations. In addition to advance reservation requests, the network must also handle regular immediate reservation requests, which are typically characterized by a pair of source and destination nodes without a specified duration. Here when a network handles an advance reservation request, the routing is done using Maxflow algorithm.

When the network handles an immediate reservation request, we first calculate the traffic load on each link on the paths. Then, we assign reservation protocols for the wavelength assignment. Two reservation protocols are discussed here namely: WAAR (Wavelength Assignment for Advance Reservation) and WAIR (Wavelength Assignment for Immediate Reservation). The goal of WAAR is to find a feasible route that can meet the specified requirements and the goal of WAIR is to find a feasible route that can maximize the duration within the range. From the simulation results we have shown that our adaptive RWA protocol attains improved throughput and bandwidth utilization.

\section{References}

1. http://en.wikipedia.org/wiki/Wavelength-division multiplexing

2. Network Simulator, http://www.isi.edu/nsnam/ns

3. JingyiHE,S.-H.GaryChan,DannyH.K.Tsang "Multicasting in WDM Networks" IEEE 2009.

4. B. Tonderayi Mangara, and F. Wilhelm Leuschner "Survivable Routing for WDM Networks: Challenges faced by the South African Service Providers" 2003.

5. P. Gurzì, A. Nowé, W. Colitti, and K. Steenhaut "Maximum Flow Based Routing and Wavelength Assignment in All-Optical Networks" 2009 IEEE.

6. Simone Cintra Chagas, Eber Huanca Cayo and Jacir Luiz Bordim "A Distributed WDM Routing and Wavelength
Assignment Protocol" IJCSNS International Journal of Computer Science and Network Security, VOL.9 No.4, April 2009.

7. Paramjeet Singh a, Ajay K. Sharmab, Shaveta Rani "Routing and wavelength assignment strategies in optical networks" 2007.

8. Vinh Trong Le $\dagger$, Xiaohong Jiang, Son Hong Ngo and Susumu Horiguchi "Dynamic RWA Based on the Combination of Mobile Agents Technique and Genetic Algorithms in WDM Networks with Sparse Wavelength Conversion" IEEE 2005.

9. X.Masip-Bruin, M.Germán, A.Castro, E.Marín-Tordera, and R.Serral-Gracià "The Minimum Coincidence Routing Approach in Wavelength-Routed Optical WDM Networks" 2009 IEEE.

10. Jorge Crichigno, Wei Shu, and Min-You Wu "Throughput Optimization and Traffic Engineering in WDM Networks Considering Multiple Metrics” IEEE ICC 2010.

11. QI Xiao-gang1, LIU Li-fang2, LIU San-yang1 “A novel algorithm for routing and wavelength assignment considering the load balancing” ISSN 2009.

12. Surendra Bhosale and Nisha Sarwade "Maximum Flow Based Load Balanced Routing Protocol for WDM Networks ", EJSR,Vol.56 No.3,pp364-375, ISSN 1450$216 \mathrm{X}$

13. Surendra Bhosale and Nisha Sarwade," Ant Based Survivable Routing and Load Balancing Technique in WDM Networks, International Review On Computers and Software (IRECOS), Italy ,vol 8(7)pp 1578-1586.

14. Rudra Dutta, Ahmed E. Kamal, and George N. Rouskas (Eds.),'Traffic Grooming for Optical Networks: Foundations, Techniques, and Frontiers', ISBN 978-0387-74517-6

15. Surendra Bhosale, Suhas Waghmare Aditya Parab, Pankaj Nikose,"Comparative analysis of different TCP variants in a wireless environment, ICECT 2011 E-ISBN 978-1-4244-8679-3

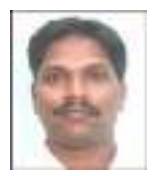

Surendra Bhosale received B.E Electrical. from Shivaji University, Kolhapur ,Maharashtra, M.E Degree in Electrical from the University of Mumbai, Maharashtra . Currently, He is pursuing the Ph.D. Degree in Electrical Engineering from the University of Mumbai, Maharashtra. Currently, $\mathrm{He}$ is an Associate Professor of Electrical Engineering Department at VJTI, Mumbai . He has more than 26 years of teaching experience. His teaching and research areas include Wireless Communications and Routing algorithms.

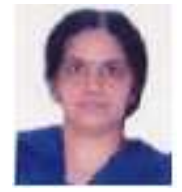

Nisha Sarwade is Associate Professor in Electrical Engineering and is a Ph.D. guide. She has obtained B.E.Degree in Electronics from Jiwai University in 1975 and M.E. Degree in solid state electronics. She obtained her Ph.D. in 1988 from University of Roorkee (Now IIT Roorkee).India. She has a vast teaching experience over 28 years and her specialization is in VLSI, Microwave, RF Communication and Nano Electronics, Nanotubes, Compound Semiconductors and Flash Memories. 